\title{
Observer-Based Control for Uncertain Nonlinear Systems Applied to Continuous Biochemical Reactors
}

\author{
Ricardo Aguilar-López $\mathbb{D}^{\mathbb{1}},{ }^{1}$ Edgar N. Tec-Caamal $\mathbb{D},{ }^{1}$ and M. Isabel Neria-González $\mathbb{D}^{2}$ \\ ${ }^{1}$ Department of Biotechnology and Bioengineering, \\ Centro de Investigación y de Estudios Avanzados del Instituto Politécnico Nacional, Mexico, Ciudad de México, \\ C.P. 07360, Mexico \\ ${ }^{2}$ Chemical and Biochemical Engineering Division, Tecnológico de Estudios Superiores de Ecatepec, Ecatepec de Morelos, \\ Estado de México, C.P. 55210, Mexico \\ Correspondence should be addressed to Ricardo Aguilar-López; raguilar@cinvestav.mx
}

Received 18 December 2019; Revised 7 July 2020; Accepted 14 July 2020; Published 30 July 2020

Academic Editor: Marco Spadini

Copyright ( $) 2020$ Ricardo Aguilar-López et al. This is an open access article distributed under the Creative Commons Attribution License, which permits unrestricted use, distribution, and reproduction in any medium, provided the original work is properly cited.

\begin{abstract}
The purpose of this paper is to present an observer-based control design with application to continuous bioreactors. For this purpose, phenomenological bioreactor models were represented by identified linear models plus unknown modelling error terms. Therefore, an uncertainty-based observer with a polynomial structure capable of estimating the unknown modelling error of the reactor representation is coupled to a linear input-output controller. The proposed methodology was evaluated in a sulphate reduction bioprocess and an acetone-butanol-ethanol (ABE) fermentation process for butanol (biofuel) production, under continuous regimes. Experimentally validated mathematical models were considered for this purpose. A theoretical framework is presented to demonstrate the corresponding closed-loop stability of the systems, and numerical simulations were carried out to corroborate the satisfactory performance of the proposed methodology.
\end{abstract}

\section{Introduction}

Biochemical reactors (BRs) are important process equipment in the transformation industry, which are currently employed in biomedical, food, fuel, and waste industries with great success [1-3]. However, despite the great progress of bioprocess engineering, several operating problems persist due to the high complex behaviour of these types of systems. From the above, processes design, operation analysis, optimization, and process control are important research issues, which can help to assure satisfactory performance of the bioreactor behaviour. Nonlinear behaviour is inherent in BR, where the state multiplicity, instabilities, and multiplicity of input, among others, make the operation of this process equipment a real challenge for process engineers [4-6]. This situation has led to intensive research in nonlinear dynamic analysis and consequently to the design of well performing nonlinear control techniques, such as predictive control, adaptive control, sliding-mode control, and neural control, among others [7-9]. The proposal of nonlinear control techniques has been very important to study the closed-loop response of BR under a theoretical framework, but unfortunately the complexity of these techniques makes difficult the real-time implementation for industrial plants. However, despite the aforementioned problems, BRs are generally controlled employing linear control designs. Among these, proportional-integral (PI) and proportional-integral-derivative (PID) controllers are widely accepted for industrial applications due to their ability to compensate most practical industrial processes. In addition, some robustness properties can be achieved with an adequate choice of controller gains [10,11]. In particular, these controllers perform well for processes with benign dynamics and modest performance requirements [12]. From the above, it is important to consider the practical importance of developing a robust controller design to achieve 
high process performance while maintaining, as much as possible, simple mathematical structures that allow potential implementation in industrial applications. In this work, the control design is composed of an uncertainty estimator coupled to a reverse dynamic feedback function to provide robustness against uncertain and neglected nonlinear dynamics. The control approach is based on simple step response models.

\section{Input-Output Identified Model}

An alternative modelling approach is to use simple input/ output models, which conserve the main dynamic characteristics of the process for control purposes in a narrow region of the state space [13]. In the classical linear control design, the feedback control design is based on the inputoutput model, which is determined from the reaction curve process, via a step disturbance of the corresponding input [13]. In particular, for chemical reactors, it is observed that the step responses are smooth, almost monotonous, and convergent in narrow stable operating regions such that it is reasonable to model the input-output response with a simple stable first-order model:

$$
G(s)=\frac{Y(s)}{U(s)}=\frac{K}{\tau s+1},
$$

where $K$ is the steady-state gain and $\tau$ is a process time constant. A state space in time domain representation of (1) can be expressed as

$$
\tau \dot{y}+y=K u .
$$

However, as is known, this linear model is only able to reproduce the system dynamic behaviour in a narrow region where the system was identified. From this, if a generalization of (2) is obtained to reproduce a wider operating region, the corresponding modelling errors of (2) must be considered to compensate the model uncertainties, as follows:

$$
\begin{gathered}
\tau \dot{y}+y+\zeta=K u, \\
\dot{\zeta}=f(y, u),
\end{gathered}
$$

where $\zeta$ represents a class of uncertain modelling error terms, which are assumed to be unknown, with an uncertain dynamic given by (4). It is assumed to be bounded, i.e., $f(y, u) \leq \Omega$.

\section{Control Design}

The main objective of the considered control law is to regulate the dynamic behaviour of the original nonlinear systems by employing representations (3) and (4). In this case, an I/O linearizing feedback approach via plant inversion is considered, so the $\mathrm{I} / \mathrm{O}$ linearizing feedback is able to provide exponentially and asymptotic closed-loop stability $[14,15]$.

The control input (5) is a linearizing controller for systems (3) and (4):

$$
u=K^{-1}(\tau \dot{y}+y+\zeta)
$$

where the desired closed-loop dynamics is suggested as a stable first-order system as follows:

$$
\dot{y}=-g_{1}\left(y-y_{s p}\right) \text {. }
$$

Therefore, the final structure of the named ideal controller is

$$
u=K^{-1}\left(-\tau g_{1}\left(y-y_{s p}\right)+y+\zeta\right) .
$$

Again, note that the control law (7) is not realizable because the term $\zeta$ is unknown.

However, as mentioned above, when nonideal conditions and uncertain terms are present, this control approach is not realizable. From this, a strategy must be proposed in order to compensate the uncertain terms and reach a realizable control design. With this purpose, an uncertainty observer-based controller must be considered.

Therefore, to avoid the above drawback, the following uncertainty observer for systems (3) and (4) is now considered:

$$
\begin{aligned}
\tau \dot{\hat{y}}+\hat{y}+\hat{\zeta} & =K \widehat{u}+g_{11}(y-\hat{y})+g_{12}(y-\hat{y})^{n}+g_{13}, \\
\dot{\bar{\zeta}} & =g_{21}(y-\hat{y})+g_{22}(y-\hat{y})^{n}+g_{23} .
\end{aligned}
$$

To analyse the convergence of the proposed observer, let us consider (3), (4), (8), and (9) to perform the dynamic equation of the estimation error $E$, by considering the Cauchy-Schwarz theorem to generate the following differential inequality:

$$
\dot{E} \leq A E-\left[\begin{array}{ll}
g_{12} & 0 \\
g_{23} & 0
\end{array}\right] E^{n}
$$

where $\mathrm{E}=\left[e_{1} e_{2}\right]^{\mathrm{T}}$ in which $e_{1}=y-\hat{y} ; e_{2}=\zeta-\widehat{\zeta}$ and $g_{i j}$ are the gains of the observer, under the following assumptions:

$$
\begin{gathered}
\|u(y, \zeta)-u(\widehat{y}, \widehat{\zeta})\| \leq L \| \mathrm{E}, \\
g_{13} \cong 0 \\
g_{21} \cong \Omega
\end{gathered}
$$

where

$$
\begin{gathered}
A=\left[\begin{array}{cc}
\tau+K^{\prime}-g_{11} & 1 \\
-g_{22} & 0
\end{array}\right], \\
K^{\prime}=K L .
\end{gathered}
$$

Note that the matrix $A$ can become a stable Hurwitz matrix by proper selection of the parameters $g_{11}$ and $g_{22}$. Moreover, inequality (10) is a Bernoulli-type ordinary differential inequality. To solve it, the following change of variable is considered:

$$
\Sigma=E^{1-n}
$$


Equation (16) transforms inequality (10) to

$$
\|\dot{\Sigma}\|-(1-n) A\|\Sigma\| \leq-(1-n) K^{\prime} .
$$

Now, the above inequality has been transformed into a linear, first-order, differential inequality, which is solved by employing the integrating factor $v=\exp ((n-1) A t)$.

$$
\|\Sigma\| \leq \nu^{-1}\left\|\Sigma_{0}\right\|+\nu^{-1} K^{\prime} A^{-1} \exp (n-1) A t,
$$

where $\Sigma_{0}$ is the initial condition of (18). Analysing the asymptotic behaviour of (18), i.e.,

$$
\lim _{t \rightarrow \infty}\|\Sigma\| \leq K^{\prime} A^{-1}
$$

or

$$
\lim _{t \rightarrow \infty}\|E\| \leq \sqrt[1-n]{K^{\prime} A^{-1}} .
$$

From the above, the nonideal controller is now given by (21), as follows:

$$
u=K^{-1}\left(-\tau g_{1}\left(y-y_{s p}\right)+y+\widehat{\zeta}\right),
$$

where $\hat{\zeta}$ is provided by observers (8) and (9).

Now, the closed-loop dynamics of systems (3) and (4) under the proposed control is

$$
\dot{y}=-g_{1}\left(y-y_{s p}\right)+\tau^{-1}(\zeta-\widehat{\zeta}) .
$$

To demonstrate the closed-loop stability of the proposed methodology and without loss of generality, assuming $y_{s p}=0$, equation (21) is solved as

$$
y=y_{0} \exp \left(-g_{1} t\right)+\exp \left(-g_{1} t\right) \int_{0}^{t} \exp \left(g_{1} \sigma\right) \tau^{-1} g_{1}^{-1}(\zeta-\widehat{\zeta}) d \sigma .
$$

Taking norms of both sides of equation (23) leads to an inequality, which is limited after boundedness assumptions:

$$
\begin{aligned}
0 \leq & \lim _{t \longrightarrow \infty} \sup \| \mathrm{y} \leq \lim _{t \longrightarrow \infty} \sup \left(\left\|y_{0}\right\| \exp \left(-g_{1} t\right)+\exp \left(-g_{1} t\right)\right. \\
& \left.\int_{0}^{t} \exp \left(g_{1} \sigma\right) \tau^{-1} g_{1}^{-1}\|\widehat{\zeta}-\zeta\| d \sigma\right) .
\end{aligned}
$$

By solving the above inequality and from the previous result, applying the convergence result for the term $\zeta-\bar{\zeta}$, the following is obtained:

$$
0 \leq \lim _{t \longrightarrow \infty} \sup \|y\| \leq \lim _{t \longrightarrow \infty} \sup \left(\tau^{-1} g_{1}^{-1} \sqrt[1-n]{K^{\prime} A^{-1}}\right) .
$$

\section{Application Examples}

The proposed control law was evaluated numerically in a sulphate reduction bioprocess and an acetone-butanolethanol (ABE) fermentation process for butanol production, under continuous regimes. Experimentally validated mathematical models were considered for this purpose. In this regard, unstructured kinetic models were used to describe the respective biosystems, due to the simplicity of the model structures. This type of model considers cells as catalysts and therefore does not consider the bioreactions that take place inside the cell. This modelling framework describes microbial growth as a function of limiting or inhibiting substrates and product formation.

As a first application case, the sulphate-reducing reactor model was based on the microbial activity of Desulfovibrio alaskensis 6SR that is an anaerobic microorganism whose growth is mainly limited by hydrogen sulphide and acetate, which are produced from the oxidation of sulphate and lactate, respectively [16]. Growth kinetics can be expressed by a double substrate function with a product inhibition term, which belongs to the kinetic model proposed by Han and Levenspiel [17], as follows.

Sulphate $\left(x_{s 1}\right)$ mass balance:

$\frac{d x_{s 1}}{d t}=D\left(x_{s 1, i n}-x_{s 1}\right)-\frac{\mu_{\max }}{Y_{2}} \frac{x_{s 1}}{k_{s}+x_{s 1}} \frac{x_{s 4}}{k_{\mathrm{lacx}}+x_{s 4}} \frac{x_{s 3}}{\left(1+x_{s 2}\right) / k_{p}}$.

Sulphide $\left(x_{s 2}\right)$ mass balance:

$\frac{d x_{s 2}}{d t}=-D x_{s 2}+r_{\operatorname{maxsh}} \frac{x_{s 1}}{k_{\text {ssh }}+x_{s 1}} \frac{x_{s 4}}{k_{\text {lacsh }}+x_{s 4}} \frac{1}{\left(1+x_{s 2}\right) / k_{p}} x_{s 3}$.

Biomass $\left(x_{s 3}\right)$ mass balance:

$\frac{d x_{s 3}}{d t}=-D x_{s 3}+\frac{\mu_{\max }}{Y_{2}} \frac{x_{s 1}}{k_{s}+x_{s 1}} \frac{x_{s 4}}{k_{\mathrm{lacx}}+x_{s 4}} \frac{1}{\left(1+x_{s 2}\right) / k_{p}} x_{s 3}$.

Lactate $\left(x_{s 4}\right)$ mass balance:

$\frac{d x_{s 4}}{d t}=D\left(x_{s 4, i n}-x_{s 1}\right)-\frac{r_{\text {maxact }}}{Y_{1}} \frac{x_{s 1}}{k_{\text {actso }}+x_{s 1}} \frac{x_{s 4}}{k_{\text {lacact }}+x_{s 4}} x_{s 3}$.

Acetate $\left(x_{s 5}\right)$ mass balance:

$\frac{d x_{s 5}}{d t}=-D x_{s 5}+r_{\text {maxact }} \frac{x_{s 1}}{k_{\text {actco }}+x_{s 1}} \frac{x_{s 4}}{k_{\text {lacct }}+x_{s 4}} \frac{1}{\left(1+x_{s 2}\right) / k_{p}} x_{s 3}$,

where $k_{d}=0.0084 \mathrm{mg} / \mathrm{L} ; k_{p}=129.77 \mathrm{mg} / \mathrm{L} ; k_{s}=4304.52 \mathrm{mg} /$ $\mathrm{L} ; k_{\text {actso }}=5038.001 \mathrm{mg} / \mathrm{L} ; k_{\text {lacact }}=3718.69 \mathrm{mg} / \mathrm{L} ; k_{\text {lach }}=36.3 \mathrm{mg} /$ $\mathrm{L} ; k_{\mathrm{lacsh}}=3577.92 \mathrm{mg} / \mathrm{L} ; k_{\mathrm{lac}} x=393.76 \mathrm{mg} / \mathrm{L} ; k_{\mathrm{ssh}}=134.12 \mathrm{mg} / \mathrm{L} ;$ $\mu_{\text {max }}=0.55 \mathrm{~h}^{-1} ; k_{\text {actco }}=0.894 ; r_{\text {maxco }}=0.132 ; k_{\text {lacco }}=4535.21 \mathrm{mg} /$ $\mathrm{L} ; \quad r_{\text {maxact }}=0.628 \mathrm{~h}^{-1} ; \quad r_{\text {maxh }}=0.033 \mathrm{~h}^{-1} ; \quad r_{\text {maxsh }}=1.495 \mathrm{~h}^{-1}$; $Y_{1}=0.825 ;$ and $Y_{2}=0.1395$.

Here, $X=\left[x_{1} x_{2} x_{3} x_{4} x_{5}\right]^{T} \in \mathbb{R}_{+}^{5}$ and $F=\left[f_{1}(X) f_{2}(X)\right.$ $\left.f_{3}(X) f_{4}(X) f_{5}(X)\right]^{T} \in \mathbb{R}_{+}^{5}$ is a nonlinear vector field whose domain belongs to $\mathscr{M}=\left\{F \in R_{+}^{5} / x_{1} \in \mathbb{R}_{1}, x_{2} \in \mathbb{R}_{2}, x_{3} \in\right.$ $\left.\mathbb{R}_{3}, x_{4} \in \mathbb{R}_{4}, x_{5} \in \mathbb{R}_{5}\right\}$, where $x_{i} \in\left[0, x_{i, \max }\right]$ for $i=1,2, \ldots$, 5 with $x_{i, \max } \in \mathbb{R}_{+}$. Besides, the named specific growth rate $\mu(\cdot): x_{2} \otimes x_{3} \otimes x_{4} \in \mathbb{R}_{+}$is a positive definite function continuously differentiable bounded by tangents planes, defined by its partial derivatives $\mu \prime=\max (\partial \mu / \partial X)<\infty$.

The second case of application relates to the production of butanol through the acetone-butanol-ethanol (ABE) fermentation process using Clostridium acetobutylicum. The 
$\mathrm{ABE}$ industrial fermentation is one of the largest biotechnology processes ever known, and its importance lies in the role of butanol as a valuable industrial solvent and its potential as a better fuel extender than ethanol [3-5]. As usual, the bioreactor model is based on the mass balance principle to describe the temporal evolution of the main state variables of the system. The equations of the bioreactor model are described as follows.

Biomass concentration:

$$
\dot{x}_{b 1}=-D x_{b 1}+\mu_{1}\left(x_{b 2}\right) \mu_{2}\left(x_{b 3}\right)^{2} x_{b 1}-k_{d} x_{b 1} \text {. }
$$

Glycerol Concentration:

$$
\dot{x}_{b 2}=D\left(x_{b 2 \text { in }}-x_{b 2}\right)-\frac{\mu_{1}\left(x_{b 2}\right) \mu_{2}\left(x_{b 3}\right)^{2} x_{b 1}}{Y_{1}} \text {. }
$$

Butanol concentration:

$$
\dot{x}_{b 3}=-D x_{b 3}-\frac{\mu_{1}\left(x_{b 2}\right) \mu_{2}\left(x_{b 3}\right)^{2} x_{b 1} Y_{2}}{Y_{1}},
$$

where

$$
\begin{gathered}
\mu_{1}\left(x_{b 2}\right)=\mu_{\max } \frac{x_{b 2}}{k_{s}+x_{b 2}} . \\
\mu_{2}\left(x_{b 3}\right)=\frac{k_{i}}{k_{i}+x_{b 3}},
\end{gathered}
$$

in which $\mu_{\max }=0.61 / \mathrm{h}, k_{s}=1.13 \mathrm{~g} / \mathrm{L}, k_{i}=7.65 \mathrm{~g} / \mathrm{L}, k_{d}=0.007$ $1 / \mathrm{h}, Y_{1}=0.57$, and $Y_{2}=3.56$ are the kinetic parameters. Note that the kinetic model considers a term of inhibition $\mu_{2}$ related to butanol concentration, as reported in the open literature [18]. This model has been experimentally validated in both batch and continuous operation regimes.

Similar to the previous bioreactor model, the state vector $X=\left[x_{1} x_{2} x_{3}\right]^{T} \in \mathbb{R}_{+}^{3}$ and $F=\left[f_{1}(X) f_{2}(X) f_{3}(X)\right]^{T} \in \mathbb{R}_{+}^{3}$ correspond to a nonlinear vector field whose domain belongs to $\mathscr{M}=\left\{F \in \mathbb{R}_{+}^{3} / x_{1} \in \mathbb{R}_{1}, x_{2} \in \mathbb{R}_{2}, x_{3} \in \mathbb{R}_{3}\right\}$, where $x_{i} \in\left[0, x_{i, \max }\right]$ for $i=1,2,3$ with $x_{i \text {,max }} \in \mathbb{R}_{+}$. Besides, the named specific growth rate $\mu(\cdot): x_{1} \otimes x_{2} \otimes x_{3} \in \mathbb{R}_{+}$is a positive definite function continuously differentiable bounded by tangents planes, defined by its partial derivatives $\mu \prime=\max (\partial \mu / \partial X)<\infty$.

All the numerical simulations were done on a PC equipped with an Intel ${ }^{\circledR}$ Core ${ }^{\circledR}$ i5 processor and $8 \mathrm{~GB}$ of RAM. The model was implemented in a script program of the MATLAB ${ }^{\circledR}$ (R2016a) software. The command ode23s from the ODE Solver library was used to solve the set of differential equations of the biochemical reactor models considering the continuous regime.

\section{Results and Discussion}

This section presents the corresponding results obtained from the numerical experiments. The continuous sulphatereducing bioreactor was simulated under the assumption of perfect mixing conditions, using the following set of initial conditions: $x_{\mathrm{s} 1,0}=6000 \mathrm{mg} / \mathrm{L}, x_{\mathrm{s} 2,0}=0.1 \mathrm{mg} / \mathrm{L}, x_{\mathrm{s} 3,0}=155 \mathrm{mg} /$ $\mathrm{L}, x_{\mathrm{s} 4,0}=5250 \mathrm{mg} / \mathrm{L}$, and $x_{\mathrm{s} 5,0}=1.5 \mathrm{mg} / \mathrm{L}$, which were considered as nominal operating conditions. In addition, to simulate different operational scenarios, the following initial conditions were also considered: $x_{\mathrm{s} 1,0}=5550 \mathrm{mg} / \mathrm{L}$, $x_{\mathrm{s} 2,0}=0.1 \mathrm{mg} / \mathrm{L}, \quad x_{\mathrm{s} 3,0}=115 \mathrm{mg} / \mathrm{L}, \quad x_{\mathrm{s} 4,0}=5150 \mathrm{mg} / \mathrm{L}$, $x_{\mathrm{s} 5,0}=1.5 \mathrm{mg} / \mathrm{L} \quad$ and $\quad x_{\mathrm{s} 1,0}=5850 \mathrm{mg} / \mathrm{L}, \quad x_{\mathrm{s} 2,0}=0.1 \mathrm{mg} / \mathrm{L}$, $x_{\mathrm{s} 3,0}=135 \mathrm{mg} / \mathrm{L}, x_{\mathrm{s} 4,0}=5550 \mathrm{mg} / \mathrm{L}$, and $x_{\mathrm{s} 5,0}=1.5 \mathrm{mg} / \mathrm{L}$. The nominal value of the dilution rate was set at $u=D=0.0351 / \mathrm{h}$. Furthermore, to include a realistic operational scenario, an external disturbance in the sulphate inlet concentration is considered as $x_{s 1 \text {,in }}=5250+1 \cdot \sin (0.1 t) \mathrm{mg} / \mathrm{L}$.

A single-input single-output (SISO) control scheme is then considered, in which the sulphate mass concentration was selected as a measured and controlled state variable. This is proposed due to the possibility of measuring the sulphate concentration online using a colorimetric technique based on spectrometric devices [19]. The input flow $(D)$ to the bioreactor is considered as the control input [20].

Then, the specific structure of the proposed controller is given by

$$
D=\frac{1}{4.87}\left(\log _{1}\left(x_{s 1}-x_{s 1, s p}\right)+x_{s 1}+\widehat{\zeta}\right)
$$

with the particular observer structure given by

$$
\begin{aligned}
\frac{d \widehat{x}_{s 1}}{d t}= & \frac{1}{170}\left(-\widehat{x}_{s 1}-\widehat{\zeta}+4.78 D+10 g_{11}\left(\widehat{x}_{s 1}-x_{s 1}\right)\right. \\
& \left.+\log _{12}\left(\widehat{x}_{s 1}-x_{s 1}\right)^{n}+0.05 g_{13}\right) \\
\frac{d \widehat{\zeta}}{d t}= & -g_{21}\left(\widehat{x}_{s 1}-x_{s 1}\right)+0.01 g_{22}\left(\widehat{x}_{s 1}-x_{s 1}\right)^{n}+0.01 g_{23}
\end{aligned}
$$

and considering the following initial conditions: $\widehat{\mathrm{x}}_{\mathrm{s} 10}=$ $5900 \mathrm{mg} / \mathrm{L}$ and $\zeta_{0}=10$.

The operation of the bioreactor was considered in an open-loop regime of up to 100 hours, where the controller was turned on. On the basis of the proposed methodology, an identification process was carried out by means of an input-output response, in which the parameters of the firstorder model are $K=4.857(\mathrm{mg} / \mathrm{L}) /\left(\mathrm{m}^{3} \mathrm{~h}\right)$ and $\tau=170$ hours. The selected values for the gains of the observer are $g_{11}=100 \mathrm{~h}^{-1} ; \quad g_{12}=1 \mathrm{~h}^{-1}(\mathrm{mg} / \mathrm{L})^{-2} ; \quad g_{13}=0.1 \mathrm{~h}^{-1} \mathrm{mg} / \mathrm{L} ;$ $g_{21}=100 \mathrm{~h}^{-1} ; g_{22}=1 \mathrm{~h}^{-1}(\mathrm{mg} / \mathrm{L})^{-2} ;$ and $g_{23}=0.1 \mathrm{~h}^{-1} \mathrm{mg} / \mathrm{L}$, with $n=3$. The control gain was selected as $g_{1}=-25 \mathrm{~h}^{-1}$. Then, to include additional disturbances in the operation of the bioreactor, a change of set point is considered. The initial set point was set at $x_{s 1, s p}=4250 \mathrm{mg} / \mathrm{L}$, but at $t=150 \mathrm{~h}$, the set point was changed to $x_{s 1, s p}=4500 \mathrm{mg} / \mathrm{L}$.

Figure 1(a) shows the behaviour of the sulphate variable in open- and closed-loop operations. The controller was turned on at $t=100$ hours for the first set point. The second set point is activated at $t=150 \mathrm{~h}$. This figure also shows the performance of the proposed uncertainty observer. At the start-up of the operation, the observer has a different initial condition of the considered real value. However, the uncertainty observer was able to reach the corresponding trajectory of the real value of the uncertain term almost immediately. This behaviour is also maintained when the controller is activated. Figure 1(b) shows the dynamic behaviour of the other uncontrolled variables, referred to as 


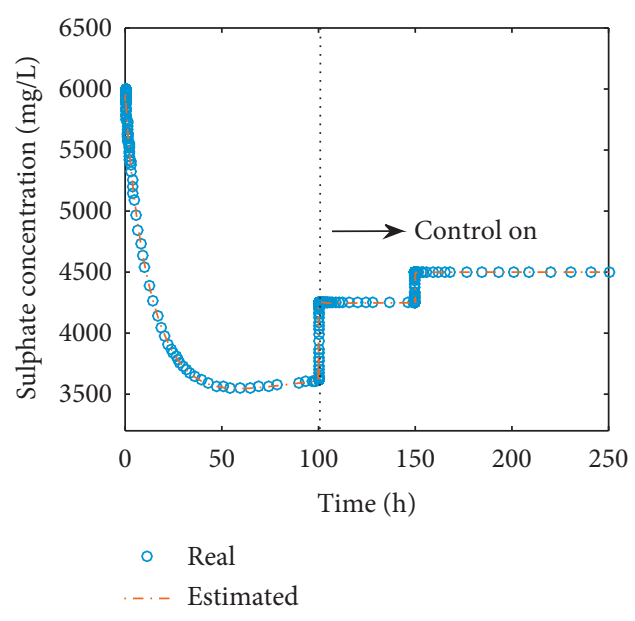

(a)

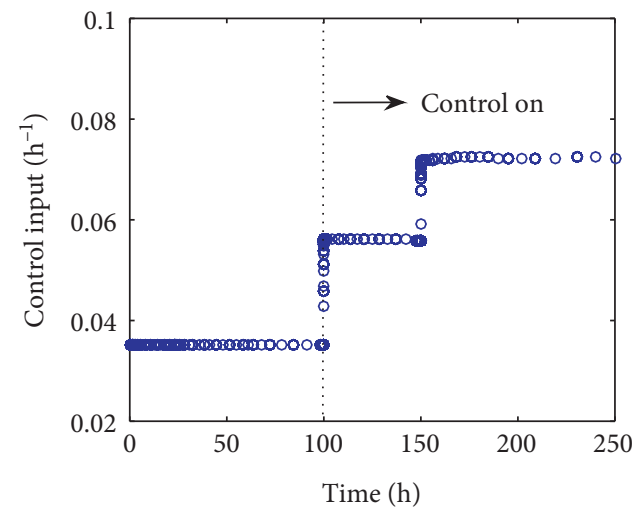

(c)

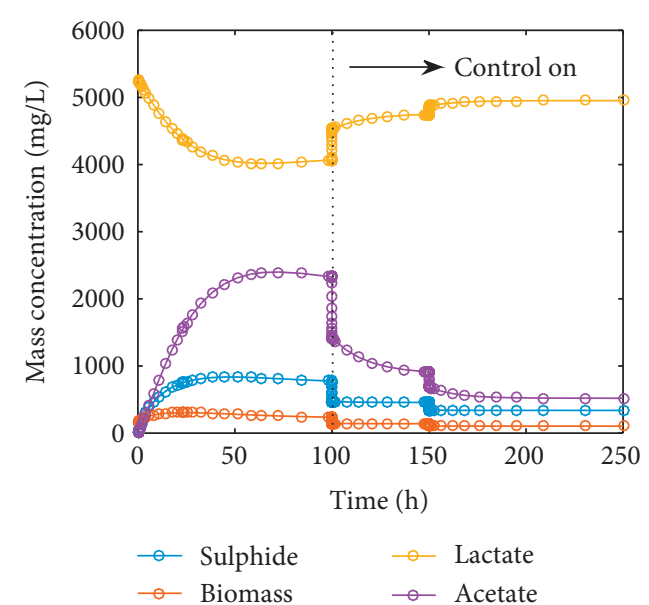

(b)

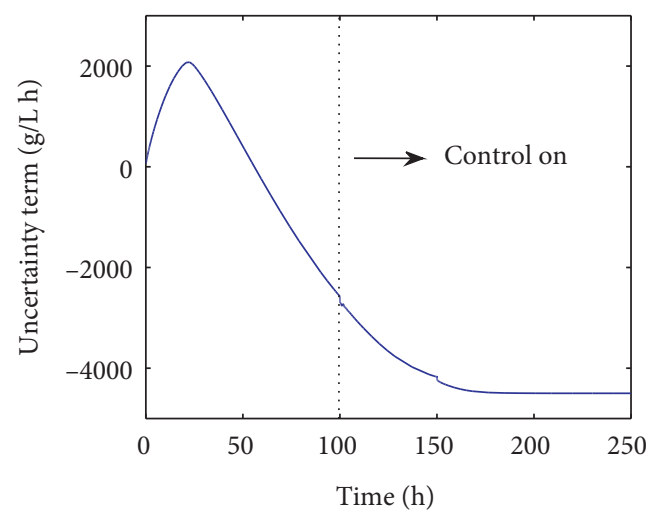

(d)

FiguRE 1: (a) Sulphate concentration as a function of time under open and closed loops. (b) Dynamics of all noncontrolled variables. (c) Control input for the two set points tested. (d) Dynamics of the uncertainty observer. The controller action is observed from $t=100 \mathrm{~h}$.

zero or inner dynamics. As can be seen, these state variables show a smooth and stable behaviour under the application of the proposed control law. The numerical results indicated that the bioreactor is stabilizable (see Figure 1(b)).

Figures 1(c) and 1(d) show the control effort and the uncertainty term of the proposed methodology. It can be seen that the control input $u$ increased from $0.035 \mathrm{~h}^{-1}$ to higher values due to the action of the controller which increases the input flow to the reactor, which in turn decreases the residence time of the reactor and the corresponding sulphate consumption until the selected set point is reached. Note that the behaviour of the control input is smooth with achievable numerical values.

Figure 2 shows a 3D phase portrait showing the behaviour of the sulphate, sulphide, and biomass variables with and without the application of the proposed control law with different initial conditions. This figure shows the sudden change of the corresponding trajectory when the closed-loop regime acts.

The second application example considers similar kind of simulation conditions as the sulphate-reducing bioreactor, with the following set of initial conditions: $x_{b 10}=0.05(\mathrm{~g} / \mathrm{L}) ; x_{b 20}=40(\mathrm{~g} / \mathrm{L}) ;$ and $x_{b 30}=0.1(\mathrm{~g} / \mathrm{L})$, which were considered as nominal operating conditions. In

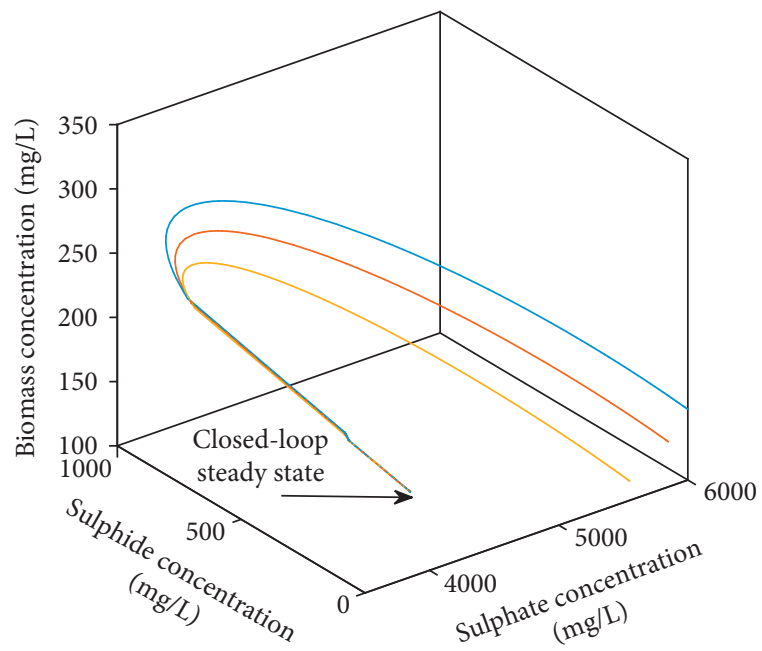

FIGURE 2: 3D phase portrait for the variables sulphate, sulphide, and biomass in open- and closed-loop systems, with different initial conditions.

addition to simulating different operational scenarios, the following initial conditions were also considered: $x_{b 10}=0.03(g / L) ; x_{b 20}=45(g / L) ; x_{b 30}=0.02(g / L) \quad$ and 


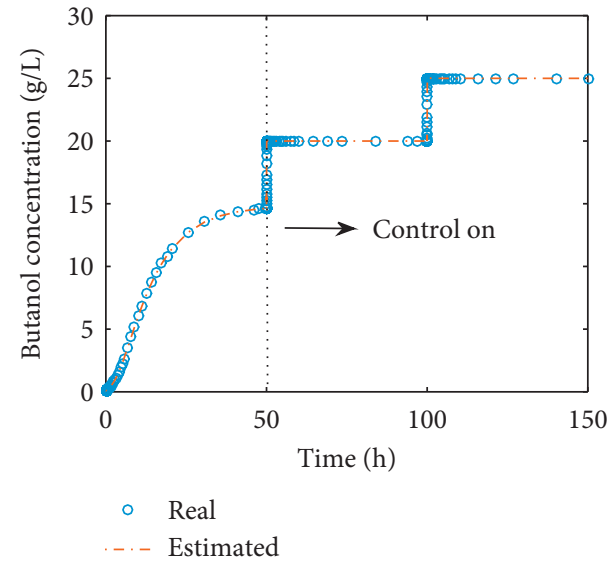

(a)

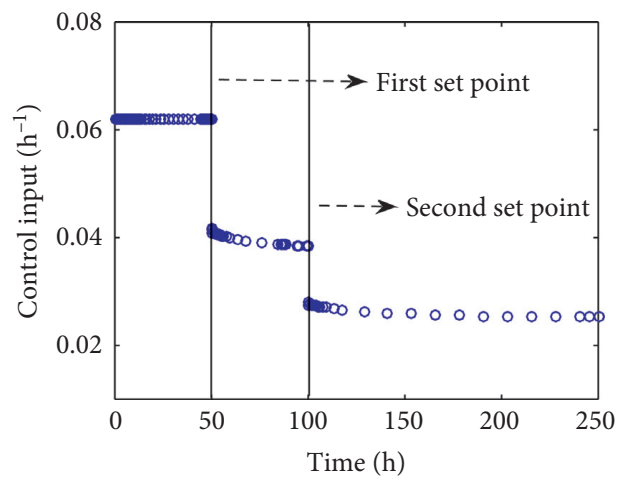

(c)

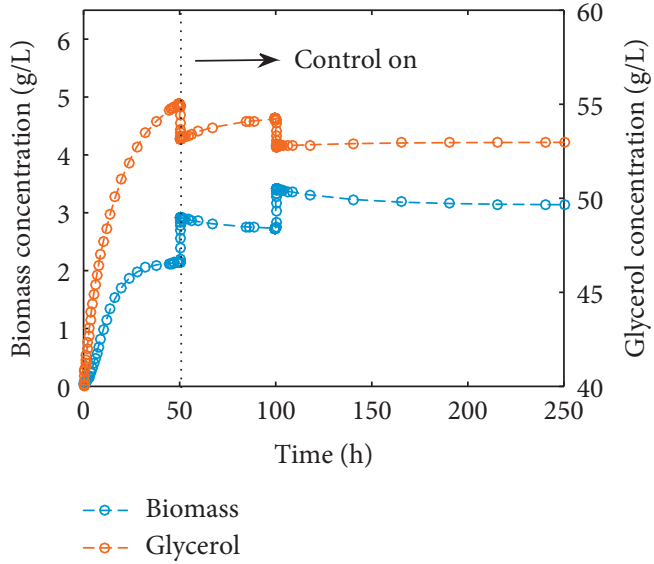

(b)

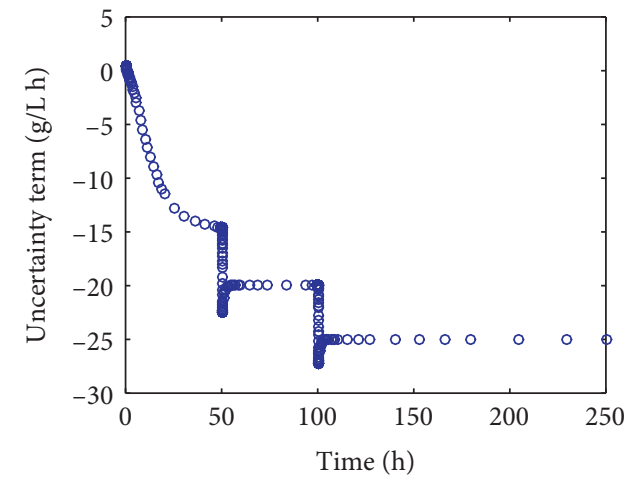

(d)

Figure 3: (a) Butanol concentration as a function of time under open and closed loops. (b) Dynamics of all noncontrolled variables. (c) Control input for the two set points tested. (d) Dynamics of the uncertainty observer. The controller action is observed from $t=50 \mathrm{~h}$.

$x_{b 10}=0.1(g / L) ; x_{b 20}=40(g / L) ; x_{b 30}=0.1(g / L) . \quad$ The nominal value of the dilution rate was set at $D=0.0621 / \mathrm{h}$. Again, SISO control scheme was considered, where the butanol mass concentration was selected as a measured and controlled state variable, since the variable can be measured online via the colorimetric technique.

The particular structure of the proposed controller is given by

$$
D=\frac{1}{2.87}\left(15 g_{1}\left(x_{b 1}-x_{b 1, s p}\right)+x_{b 1}+\widehat{\zeta}\right),
$$

with the corresponding observer structure:

$$
\begin{gathered}
\frac{\mathrm{d} \widehat{x}_{b 1}}{\mathrm{~d} t}=\frac{1}{1.5}\left(-\widehat{x}_{s 1}-\widehat{\zeta}+0.48 D+g_{11}\left(\widehat{x}_{s 1}-x_{s 1}\right)\right. \\
\left.+0.1 g_{12}\left(\widehat{x}_{s 1}-x_{s 1}\right)^{n}+0.1 g_{13}\right), \\
\frac{\mathrm{d} \widehat{\zeta}}{\mathrm{d} t}=-g_{21}\left(\widehat{x}_{s 1}-x_{s 1}\right)+0.1 g_{22}\left(\widehat{x}_{s 1}-x_{s 1}\right)^{n}+0.1 g_{23} .
\end{gathered}
$$

Here, the corresponding tuning of the gains of the observer are $g_{11}=100 \mathrm{~h}^{-1} ; g_{12}=1 \mathrm{~h}^{-1}(\mathrm{~g} / \mathrm{L})^{-2} ; g_{13}=0.1 \mathrm{~h}^{-1} \mathrm{~g} /$ $\mathrm{L} ; \quad g_{21}=100 \mathrm{~h}^{-1}(\mathrm{~g} / \mathrm{L})^{-2} ; \quad g_{22}=1 \mathrm{~h}^{-1}(\mathrm{~g} / \mathrm{L})^{-2} ; \quad$ and $g_{23}=0.1 \mathrm{~h}^{-1} \mathrm{~g} / \mathrm{L}$, with $n=3$. The control gain was selected as

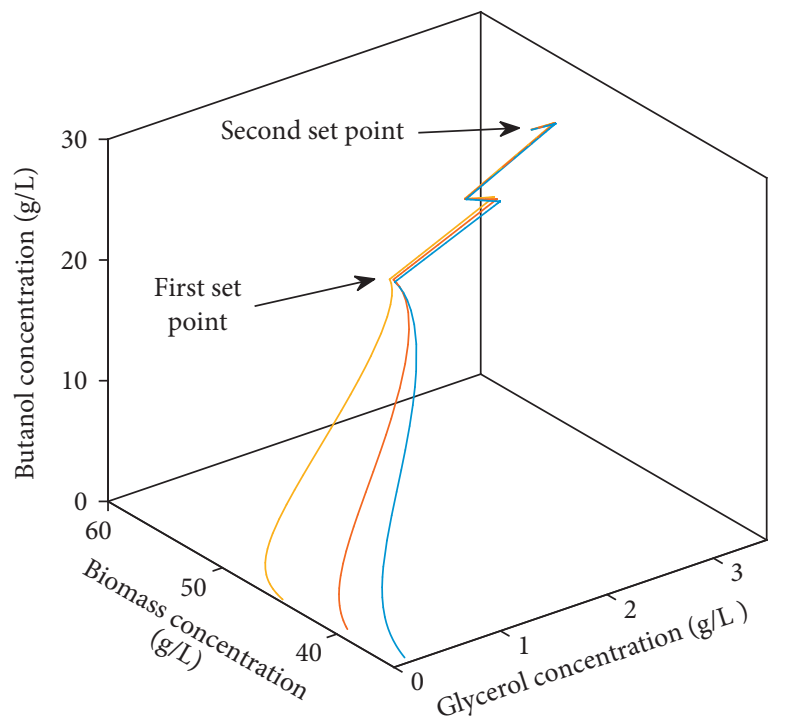

FIgURE 4: 3D phase portrait for the variables biomass, glycerol, and butanol in open- and closed-loop systems, with different initial conditions.

$g_{1}=10 \mathrm{~h}^{-1}$. Furthermore, to include additional disturbances in the operation of the bioreactor, a set point change was 
considered. The initial set point was selected as $x_{s 1, s p}=20(\mathrm{~g} / \mathrm{L})$, and at $t=100 \mathrm{~h}$, the set point is changed to $x_{s 1, s p}=25(\mathrm{~g} / \mathrm{L})$. The controller was turned on at $t=50 \mathrm{~h}$.

Figure 3(a) shows the behaviour of the butanol variable in open- and closed-loop operations. Once again, the uncertainty observer was able to rapidly reach the trajectory of the real value of the uncertain variable in open and closed loops. Figure 3(b) depicts the dynamics of the biomass and glycerol that are the noncontrolled variables, which showed a stable behaviour under the application of the proposed controller. Figures 3(c) and 3(d) show the control effort and the uncertainty term for both set points. It can be observed that the controller decreases the input flow to the reactor, which in turn increases the residence time of the reactor, allowing to increase the glycerol consumption and thus obtaining higher butanol production until the selected set point is reached. Once again, the control input showed achievable numerical values. Figure 4 shows a $3 \mathrm{D}$ phase portrait of the dynamics of the three variables with and without the application of the proposed control law with different initial conditions, where it is possible to observe the trajectory changes when the controller was activated, taking the process to different steady-state points.

\section{Concluding Remarks}

In this work, a class of uncertainty observer-based inputoutput controller for regulation purposes with application to continuous bioreactors is presented. The proposed uncertainty observer contains a polynomial structure of the measured output injection. The convergence of the proposed observer is demonstrated via the analysis of the dynamic equation of the estimation error, where an asymptotic convergence is depicted around an open ball with radius equal to $R=\sqrt[1-n]{K^{\prime} A^{-1}}$. A satisfactory performance of the observer was observed via numerical simulations both in open-loop and closed-loop operational regimes. The observer was coupled with a linearizing I/O controller, where the closed-loop stability is analysed by means of the dynamic equation of the regulation error, predicting an asymptotic convergence of the control output, which was also shown by numerical simulations, where two application cases related with continuous biochemical reactors are considered with successful results.

\section{Data Availability}

The data used to support the findings of the study are available from the corresponding author upon request.

\section{Conflicts of Interest}

The authors declare that they have no conflicts of interest.

\section{References}

[1] J. López, V. F. Cataldo, M. Peña et al., "Build your bioprocess on a solid strain- $\beta$-carotene production in recombinant Saccharomyces cerevisiae," Frontiers in Bioengineering and Biotechnology, vol. 7, 2019.
[2] R. M. Lindeque and J. M. Woodley, "Reactor selection for effective continuous biocatalytic production of pharmaceuticals," Catalysts, vol. 9, 2019.

[3] E. N. Tec-Caamal, A. Jiménez-González, S. A. MedinaMoreno, and M. A. Lizardi-Jiménez, "Production of an oildegrading bacterial consortium in an airlift bioreactor: insights into the mass transfer of the oil and oxygen," Chemical Engineering Science, vol. 192, pp. 507-515, 2018.

[4] L. F. Calderón-Soto, E. J. Herrera-López, G. Lara-Cisneros, and R. Femat, "On unified stability for a class of chemostat model with generic growth rate functions: maximum yield as control goal," Journal of Process Control, vol. 77, pp. 61-75, 2019.

[5] L. F. Calderón-Soto, J. M. Méndez-González, E. J. HerreraLópez, C. Ghommidh, and R. Femat, "Determination of experimental and mathematical oscillatory conditions for Zymomonas mobilis with different death rates for viable and VBNC cells," Chemical Engineering Research and Design, vol. 123, pp. 407-415, 2017.

[6] R. V. Gomez-Acata, G. Lara-Cisneros, R. Femat, and R. Aguilar-López, "On the dynamic behaviour of a class of bioreactor with non-conventional yield coefficient form," Revista Mexicana de Ingeniería Química, vol. 14, no. 1, 2015.

[7] N. Khanduja and B. Bhushan, "Control system design and performance analysis of PID and IMC controllers for continuous stirred tank reactor (CSTR)," Journal of Control \& Instrumentation, vol. 10, no. 1, pp. 16-22, 2019.

[8] A. Sinha and R. K. Mishra, "Control of a nonlinear continuous stirred tank reactor via event triggered sliding modes," Chemical Engineering Science, vol. 187, pp. 52-59, 2018.

[9] R. Aguilar-López, E. N. Tec-Caamal, and J. L. Mata-Machuca, "Dynamic optimal control for a class of nonlinear system," in Advances in Engineering Research, V. M. Petrova, Ed., Nova Science Publisher, New York, NY, USA, 2019.

[10] N. Khanduja and B. Bhushan, "CSTR control using IMC-PID, PSO-PID, and hybrid BBO-FF-PID controller," in Applications of Artificial Intelligence Techniques in Engineering, H. Malik, S. Srivastava, Y. Sood, and A. Ahmad, Eds., vol. 697, Springer, Singapore, 2019.

[11] W.-D. Chang and S.-P. Shih, "PID controller design of nonlinear systems using an improved particle swarm optimization approach," Communications in Nonlinear Science and Numerical Simulation, vol. 15, no. 11, pp. 3632-3639, 2010.

[12] A. O'Dwyer, Handbook of PI and PID Controller Tuning Rules, Imperial College Press, London, UK, 2009.

[13] B. A. Ogunnaike and W. H. Ray, Process Dynamics, Modeling and Control, Oxford University Press, New York, NY, USA, 1994.

[14] J. Alvarez-Ramírez and R. Suarez, "Stabilization of a class of linear time-varying systems via modeling error compensation," IEEE Transactions on Automatic Control, vol. 45, no. 4, 2000.

[15] J. A. Romero-Bustamante, J. G. Moguel-Castañeda, H. Puebla, and E. Hernández-Martínez, "Robust cascade control for chemical reactors: an approach based on modeling error compensation," International Journal of Chemical Reactor Engineering, vol. 15, Article ID 20170082, 2017.

[16] O. Levenspiel, "The monod equation: a revisit and a generalization to product inhibition situations," Biotechnology and Bioengineering, vol. 22, no. 8, pp. 1671-1687, 1980.

[17] K. Han and O. Levenspiel, "Extended monod kinetics for substrate, product, and cell inhibition," Biotechnology and Bioengineering, vol. 32, no. 4, pp. 430-447, 1988. 
[18] H. I. Velázquez-Sánchez and R. Aguilar-López, "Novel kinetic model for the simulation analysis of the butanol productivity of Clostridium acetobutylicum ATCC 824 under different reactor configurations," Chinese Journal of Chemical Engineering, vol. 26, no. 4, pp. 812-821, 2018.

[19] A. Roy, B. K. Das, and J. Bhattacharya, "Development and validation of a spectrophotometric method to measure sulfate concentrations in mine water without interference," Mine Water and the Environment, vol. 30, no. 3, pp. 169-174, 2011.

[20] S. Liu, Bioprocess Engineering: Kinetics, Sustainability, and Reactor Design, Elsevier, Alpharetta, GA, USA, 2017. 